\title{
Point-of-care ultrasonography improves the diagnosis of splenomegaly in hospitalized patients
}

\author{
Andrew P. J. Olson ${ }^{1,2^{*}}$, Bernard Trappey ${ }^{1,2}$, Michael Wagner ${ }^{3}$, Michael Newman ${ }^{1,4}$, L. James Nixon ${ }^{1,2}$ \\ and Daniel Schnobrich ${ }^{1,2}$
}

\begin{abstract}
Background: It is important to detect splenomegaly as it can have important diagnostic implications. Previous studies, however, have shown that the traditional physical examination is limited in its ability to rule in or rule out splenomegaly.
\end{abstract}

Objective: To determine if performing point-of-care ultrasonography (POCUS) in addition to the traditional physical examination improves the sensitivity and specificity for diagnosing splenomegaly.

Methods: This was a prospective trial of diagnostic accuracy. Physical and sonographic examinations for splenomegaly were performed by students, residents and attending physicians enrolled in an ultrasound training course. Participants received less than $1 \mathrm{~h}$ training for ultrasound diagnosis of splenomegaly. The findings were compared to radiographic interpretation of gold standard studies.

Setting/patients: Hospitalized adult patients at an academic medical center without severe abdominal pain or recent surgery who had abdominal CT, MRI or ultrasound performed within previous $48 \mathrm{~h}$.

Results: Thirty-nine subjects were enrolled. Five patients had splenomegaly $(12.5 \%)$. The physical examination for splenomegaly had a sensitivity of $40 \%(95 \% \mathrm{Cl} 12-77 \%)$ and specificity of $88 \%(95 \% \mathrm{Cl} 74-95 \%)$ while physical examination plus POCUS had a sensitivity of $100 \%$ (95 \% Cl 57-100\%) and specificity of $74 \%$ (95\% Cl 57-85\%). Physical examination alone for splenomegaly had an LR+ of 3.4 (95\% Cl 0.83-14) and LR- of $0.68(95 \% \mathrm{Cl} 0.33-1.41)$; for physical exam plus POCUS the LR+ was 3.8 (2.16-6.62) and LR- was 0.

Conclusions: Point-of-care ultrasonography significantly improves examiners'sensitivity in diagnosing splenomegaly. Keywords: Point-of-care ultrasound, Diagnosis, Splenomegaly, Sensitivity, Specificity, Physical examination

\section{Background}

Splenomegaly is an important clinical finding that can have significant diagnostic implications. Its presence may be an important clue to malignancy, infections, or inflammatory conditions. Conversely, the absence of splenomegaly can also be an important finding and influence the diagnostic evaluation.

\footnotetext{
*Correspondence: olso5714@umn.edu

1 Department of Medicine, University of Minnesota Medical School, 420

Delaware St, MMC 741, Minneapolis, MN 55455, USA

Full list of author information is available at the end of the article
}

Historically, the reference standard for evaluating diagnostic accuracy of tests for splenomegaly has been splenic scintigraphy [1] or ultrasonography [2-4], but more recently computed tomography has also been used as a reference standard. The physical examination [3] for splenomegaly includes palpation, percussion by Nixon's method [5], percussion by Castell's method [6], and percussion of Traube's space [7]. These commonly used methods have sensitivity ranging from 11 to $85 \%$ and specificity from 32 to $99 \%$.

Point-of-care ultrasonography (POCUS) involves the acquisition and interpretation of a narrow set of simple,

\section{贷 Springer}


often dichotomous, findings by a provider at the patient's bedside [8]. Emergency physicians were the first in the United States to show that POCUS markedly improved the detection of important findings such as intra-abdominal fluid [9]. Further studies have demonstrated that focused training for a broad number of ultrasound skills during residency was feasible [10-13]. More recently, POCUS has become increasingly common in fields such as internal medicine and family medicine. The purpose of our study was to evaluate the use of POCUS in addition to the traditional physical exam for detection of splenomegaly. We hypothesized that, with limited training, point-of-care ultrasonography would outperform the traditional physical examination.

\section{Methods}

Ultrasonographic and physical exam assessments for splenomegaly were performed on a convenience sample of patients admitted to the Hospital Medicine and Hematology/Oncology services at the University of Minnesota Medical Center between July 2013 and March 2015. One investigator screened the medical records of patients to identify those meeting the following criteria: (1) 18 years of age or older; (2) capable of giving informed consent; (3) lack of moderate-to-severe abdominal pain; (4) lack of abdominal surgeries in the last 30 days; (5) CT, MRI, or ultrasound of the abdomen had been performed in the course of usual clinical care within the last $48 \mathrm{~h}$. Eligible patients were then approached and those who consented underwent ultrasound and physical exams as described below (Fig. 1).

Traditional physical examinations and physical examinations plus POCUS were performed by medical students, residents and attending physicians (examiners). The examiners included internal medicine residents and hospitalists participating in an ultrasound course at the University of Minnesota Medical School [13] and third and fourth year medical students in an advanced physical diagnosis elective course. The training for examiners included a 15-min didactic presentation on how to measure the spleen along with practice on a healthy model 2-3 times over the course of several days.

Examiners were randomly assigned to perform either the traditional examination alone or traditional exam in conjunction with POCUS. For each patient enrolled, one traditional physical examination and one physical examination plus POCUS were performed by different examiners in succession (neither examiner had knowledge of the others' findings). Sonographic examinations were performed using a portable ultrasound machine

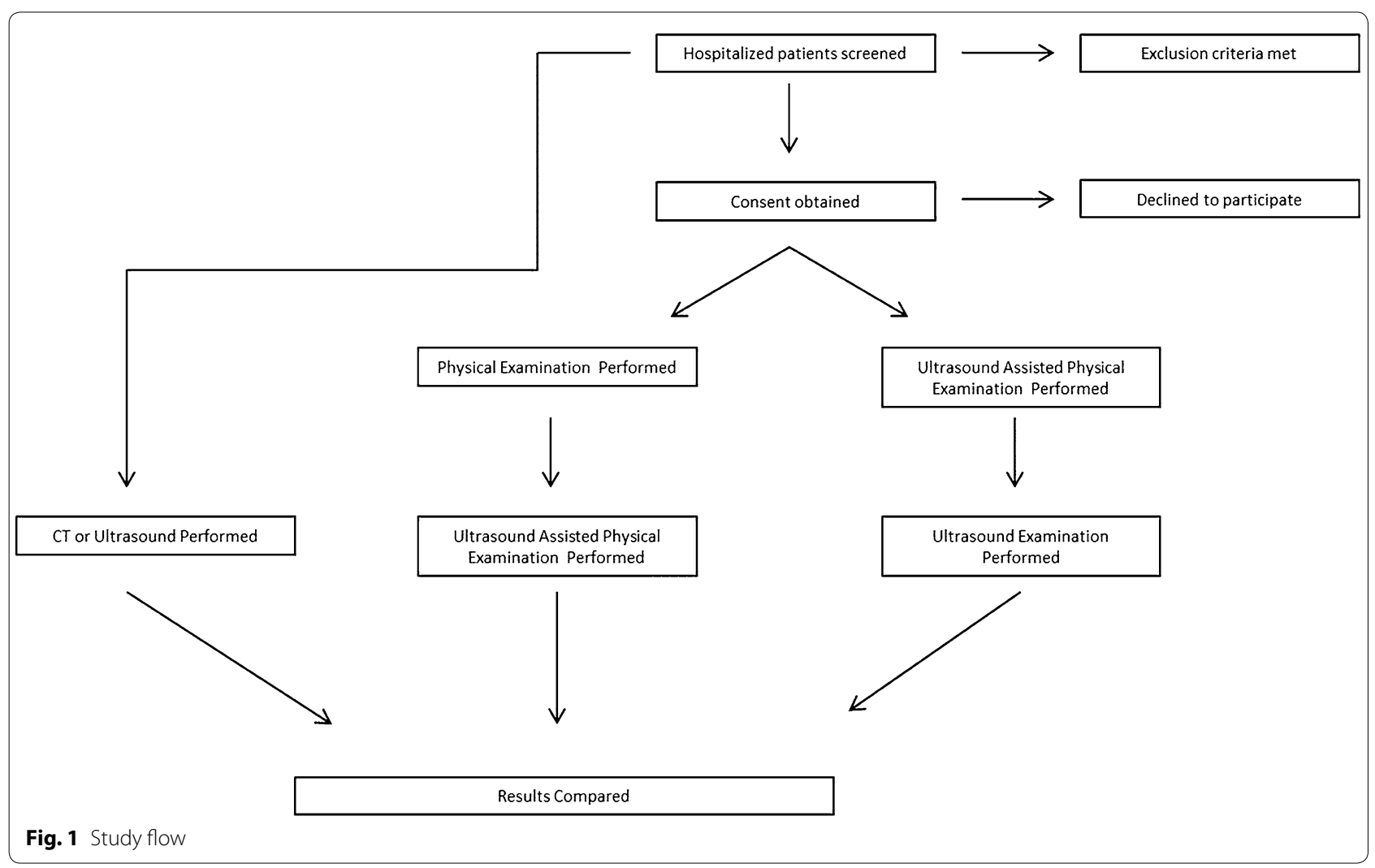


(NanoMaxx, Sonosite, Washington, USA) phased array or abdominal probe.

Physical examinations of spleen size were performed according to the examiners' usual clinical practice; no history-taking was allowed, and patients were not required to be fasting. Sonographic examinations of the spleen were performed by placing the transducer in the left upper quadrant and examining the entire spleen. The largest anteroposterior dimension of the spleen was identified and measured. Splenomegaly was defined as an anteroposterior dimension $>13 \mathrm{~cm}$ [14]. At a later time another investigator recorded whether or not splenomegaly was noted in the formal radiology report and this information used as the reference standard for the study. All clinicians performing examinations were blinded to the patients' medical history, medical record, and formal radiology results.

This study was approved by the University of Minnesota Institutional Review Board.

\section{Statistical analysis}

Data were entered into a secure database (RedCap) [15]. Sensitivities, specificities, likelihood ratios, and confidence intervals were calculated for physical examination and for physical examination plus POCUS, with the formal radiological study serving as the reference standard. Fisher's exact test was used to calculate statistical significance for diagnostic tests in $2 \times 2$ contingency tables (Graphpad, La Jolla, CA, USA).

\section{Results}

A total of 39 subjects were enrolled in the study. Five patients had splenomegaly by the reference standard (12.5\%). Seven medical students, 16 residents and 1 attending physician performed examinations during the study.

For splenomegaly, physical examination had a sensitivity of $40 \%$ (95\% CI 12-77\%) and specificity $88 \%$ (95\% CI 74-95\%) ( $p=0.161)$ while physical examination plus POCUS had a sensitivity of $100 \%$ (95 \% CI 57-100\%) and specificity $74 \%(95 \%$ CI $57-85 \%)(p=0.003)$. For physical examination alone for splenomegaly, the LR+ was 3.4 (95\% CI 0.83-14) and LR- 0.68 (95\% CI $0.33-1.41)$; for physical exam plus POCUS the LR+ was 3.8 (2.16-6.62) and LR - was 0 (Table 1).

\section{Conclusions}

We show that, in keeping with previous studies, the ability of the traditional physical examination to detect splenomegaly is limited. Performing point-of-care ultrasound in addition to the traditional physical examination improves the sensitivity for splenomegaly. In patients for whom the presence of splenomegaly would significantly alter the differential diagnosis, clinicians should use POCUS in addition to their traditional exam, as a negative exam effectively rules out splenomegaly.

This study has a number of important limitations. First, the sample size is relatively small. Second, this was a single-center study at an academic medical center with a significant number of end-stage liver disease patients, and thus there may be some availability bias [16], though this should have affected the physical and ultrasound exams equally. In addition, no history was taken and history-taking influences the accuracy of the physical examination [17]. Finally, the radiologist interpreting the images may not have used the most accurate measurement methods in his or her interpretation of the radiologic study, leading to a suboptimal reference standard.

It should also be noted that while the sensitivity of POCUS was much higher than the physical exam alone, POCUS was not more specific in this study. It is important that clinicians performing this exam are aware of the limited specificity of this exam to guard against overconfidence in applying a positive result. It follows that POCUS should be used to detect splenomegaly only when this is a clinically important question for a given patient; its use as part of a standard examination for every patient or as a screening test would result in many false positives. Presence of splenomegaly on POCUS

Table 1 Traditional physical examination and ultrasound-assisted physical examination findings for splenomegaly

\begin{tabular}{|c|c|c|c|c|c|c|c|c|}
\hline Condition & $\begin{array}{l}\text { Reference } \\
\text { standard }\end{array}$ & Test & $n$ (patients) & $\begin{array}{l}\text { Sensitivity } \\
(95 \% \text { CI) [TP/ } \\
\text { FN + TP] }\end{array}$ & $\begin{array}{l}\text { Specificity } \\
(95 \% \text { CI) [TN/ } \\
\text { TN + FP] }\end{array}$ & $p$ value* & $\mathrm{LR}+(95 \% \mathrm{Cl})$ & LR- $(95 \% \mathrm{Cl})$ \\
\hline \multirow[t]{2}{*}{ Splenomegaly } & CT or US & $\begin{array}{l}\text { Physical examina- } \\
\text { tion alone }\end{array}$ & 39 & $\begin{array}{l}0.40(0.12-.77) \\
{[2 / 5]}\end{array}$ & $\begin{array}{l}0.88(0.73-0.95) \\
{[30 / 34]}\end{array}$ & 0.161 & $\begin{array}{l}3.40(0.827- \\
13.98)\end{array}$ & $0.68(0.33-1.41)$ \\
\hline & & $\begin{array}{l}\text { Physical } \\
\text { exam + PoCus }\end{array}$ & & $1(0.57-1)[5 / 5]$ & $\begin{array}{l}0.74(0.57-0.85) \\
{[25 / 34]}\end{array}$ & 0.003 & $3.78(2.16-6.61)$ & 0 \\
\hline
\end{tabular}

$T P$ true positive, $F P$ false positive, $F N$ false negative, $T N$ true negative, $L R+$ positive likelihood ratio, $L R-$ negative likelihood ratio, $C T$ computed tomography, US ultrasound

* $p$ value for Fisher's exact test 
should be confirmed with standard radiologic tests if the finding has clinical importance.

In conclusion, the performance of point-of-care ultrasonography in addition to the traditional physical examination improves students', residents', and attending physicians' ability to detect splenomegaly. Given the relative ease of training for POCUS, one may consider adding this as a standard part of the clinical evaluation for splenomegaly.

\section{Abbreviations}

POCUS: point-of-care ultrasonography; LR (+): positive likelihood ratio; LR (-): negative likelihood ratio.

\section{Authors' contributions}

AO conceived the project, wrote the IRB applications and protocols, assisted in data collection, performed the analysis, and drafted the manuscript. BT assisted in data collection and analysis and helped draft and edit the manuscript. MW and MN assisted in data collection and analysis. LN assisted in the study design as well as the analysis and drafting of the manuscript. DS assisted in study conception, assisted in data collection, assisted with analysis, and helped draft and edit the manuscript. All authors read and approved the final manuscript.

\section{Author details}

1 Department of Medicine, University of Minnesota Medical School, 420 Delaware St, MMC 741, Minneapolis, MN 55455, USA. ${ }^{2}$ Department of Pediatrics, University of Minnesota Medical School, Minneapolis, MN, USA. ${ }^{3}$ Department of Medicine, Weill Cornell Medical College, New York, NY, USA. ${ }^{4}$ Minneapolis VA Medical Center, Minneapolis, MN, USA.

\section{Acknowledgements}

The authors wish to thank the staff of the Simulation PeriOperative Resource for Training and Learning (SimPortal) at the University of Minnesota for their assistance with the study. The project described was supported by Award Number UL1TR000114 from the National Center for Advancing Translational Sciences (NCATS) of the National Institutes of Health $(\mathrm{NIH})$. The content is solely the responsibility of the authors and does not necessarily represent the official views of the National Center for Research Resources or the National Institutes of Health.

\section{Compliance with ethical guidelines}

\section{Competing interests}

$\mathrm{AO}$ and DS have been loaned ultrasound equipment by Sonosite for another study. Sonosite has no role in the design, implementation, or analysis of this or the other study.

Received: 25 June 2015 Accepted: 17 August 2015

Published online: 17 September 2015

\section{References}

1. Skrainka B, Stahlhut J, Fulbeck CL, Knight F, Holmes RA, Butt JH (1986) Measuring liver span. Bedside examination versus ultrasound and scintiscan. J Clin Gastroenterol 8(3 Pt 1):267-270

2. Koga T, Morikawa Y (1975) Ultrasonographic determination of the splenic size and its clinical usefulness in various liver diseases. Radiology 115(1):157-161

3. Grover SA (1993) Does This patient have splenomegaly? JAMA J Am Med Assoc 270(18):2218

4. Benter T, Klühs L, Teichgräber U (2011) Sonography of the spleen. J Ultrasound Med 30(9):1281-1293

5. Nixon RK (1954) The Detection of splenomegaly by percussion. N Engl J Med 250(4):166-167

6. Castell DO (1967) The spleen percussion sign: A useful diagnostic technique. Ann Intern Med 67(6):1265-1267

7. Barkun AN, Camus M, Meagher Tet al (1989) Splenic enlargement and Traube's space: how useful is percussion? Am J Med 87(5):562-566

8. Moore CL, Copel JA (2011) Point-of-care ultrasonography. N Engl J Med 364(8):749-757

9. Patel NY, Riherd JM (2011) Focused assessment with sonography for trauma: methods, accuracy, and indications. Surg Clin North Am 91(1):195-207

10. Chisholm CB, Dodge WR, Balise RR, Williams SR, Gharahbaghian L, Beraud A-S (2013) Focused cardiac ultrasound training: how much is enough? J Emerg Med 44(4):818-822

11. Jones AE, Tayal VS, Kline JA (2003) Focused training of emergency medicine residents in goal-directed echocardiography: a prospective study. Acad Emerg Med 10(10):1054-1058

12. Ruddox V, Stokke TM, Edvardsen T et al (2013) The diagnostic accuracy of pocket-size cardiac ultrasound performed by unselected residents with minimal training. Int J Cardiovasc Imaging 29(8):1749-1757

13. Schnobrich DS, Olson APJ, Broccard A, Duran-Nelson A (2013) Feasibility and acceptability of a structured curriculum in teaching procedural and basic diagnostic ultrasound skills to internal medicine residents. J Grad Med Educ 5(3):493-573

14. Hosey RG (2006) Ultrasound assessment of spleen size in collegiate athletes. Br J Sports Med 40(3):251-254

15. Harris PA, Taylor R, Thielke R, Payne J, Gonzalez N, Cond JG (2009) Research electronic data capture (REDCap) — A metadata-driven methodology and workflow process for providing translational research informatics support. J Biomed Inform 42(2):377-381

16. Croskerry P (2003) The importance of cognitive errors in diagnosis and strategies to minimize them. Acad Med 78(8):775-780

17. Sibbald M, Cavalcanti RB (2011) The biasing effect of clinical history on physical examination diagnostic accuracy. Med Educ 45(8):827-834

\section{Submit your manuscript to a SpringerOpen ${ }^{\circ}$ journal and benefit from:}

- Convenient online submission

- Rigorous peer review

- Immediate publication on acceptance

- Open access: articles freely available online

- High visibility within the field

- Retaining the copyright to your article

Submit your next manuscript at springeropen.com 